\title{
Serum Markers and Intestinal Mucosal Injury in Chronic Gastrointestinal Ischemia
}

\author{
Désirée van Noord • Peter B. F. Mensink • Robert J. de Knegt • \\ Martine Ouwendijk · Jan Francke • Anneke J. van Vuuren • \\ Bettina E. Hansen · Ernst J. Kuipers
}

Received: 19 October 2009/Accepted: 14 June 2010/Published online: 15 July 2010

(C) The Author(s) 2010. This article is published with open access at Springerlink.com

\begin{abstract}
Background Diagnosing chronic gastrointestinal ischemia (CGI) is a challenging problem in clinical practice. Serum markers for CGI would be of great diagnostic value as a non-invasive test method.

Aims This study investigated serum markers in patients with well-defined ischemia. Furthermore, intestinal mucosal injury was also evaluated in CGI patients.

Methods Consecutive patients suspected of CGI were prospectively enrolled and underwent a diagnostic work-up consisting of gastrointestinal tonometry and either CT or MR angiography. Blood samples for analysis of intestinal fatty acid-binding protein (I-FABP), D-dimer, lactate dehydrogenase (LDH), leucocyte counts, C-reactive protein (CRP), and L-lactate were drawn before and after a standard meal. Intestinal mucosal injury was assessed with glutamine, citrulline and arginine in blood samples and
\end{abstract}

D. van Noord $(\bowtie)$ · P. B. F. Mensink · R. J. de Knegt .

M. Ouwendijk - J. Francke - A. J. van Vuuren .

B. E. Hansen · E. J. Kuipers

Department of Gastroenterology and Hepatology,

Erasmus MC-University Medical Center, 's-Gravendijkwal

230, 3015 CE Rotterdam, The Netherlands

e-mail: d.vannoord@erasmusmc.nl

B. E. Hansen

Department of Biostatistics, Erasmus MC_-University Medical

Center, Rotterdam, The Netherlands

E. J. Kuipers

Department of Internal Medicine, Erasmus MC-University

Medical Center, Rotterdam, The Netherlands compared to a sugar absorption test (SAT). Test reproducibility was validated in healthy subjects.

Results Forty patients and nine healthy subjects were included. Ischemia was diagnosed in 32 patients $(80 \%)$. I-FABP, leucocyte counts, LDH, CRP, glutamine, citrulline, arginine and SAT levels did not differ between patients with and without ischemia. L-lactate concentration showed a significant elevation in ischemia patients as compared to non-ischemia patients. In ischemia patients, D-dimer levels showed a significant elevation postprandially as compared to D-dimer levels at baseline. However, these ischemia patients did not show intestinal mucosal injury.

Conclusions I-FABP, leucocyte counts, LDH and CRP levels are not clinically useful for the diagnosis of CGI. However, postprandial rises in L-lactate and D-dimer serum levels can serve as non-invasive indicators of CGI.

Keywords Early serum markers - Late serum markers . Chronic gastrointestinal ischemia .

Intestinal mucosal injury $\cdot \mathrm{D}$-dimer $\cdot$ L-lactate

$\begin{array}{ll}\text { Abbreviations } \\ \text { CA } & \text { Celiac artery } \\ \text { CGI } & \text { Chronic gastrointestinal ischemia } \\ \text { CRP } & \text { C-reactive protein } \\ \text { CTA } & \text { Computed tomography angiography } \\ \text { I-FABP } & \text { Intestinal fatty acid binding protein } \\ \text { IMA } & \text { Inferior mesenteric artery } \\ \text { LDH } & \text { Lactate dehydrogenase } \\ \text { MRA } & \text { Magnetic resonance angiography } \\ \text { NOMI } & \text { Non-occlusive mesenteric ischemia } \\ \text { SAT } & \text { Sugar absorption test } \\ \text { SMA } & \text { Superior mesenteric artery } \\ \text { TM } & \text { 24-hour gastric and jejunal tonometry }\end{array}$




\section{Introduction}

Chronic gastrointestinal ischemia (CGI) is a diagnostic challenge. There is no single, simple test with adequate predictive value to diagnose or exclude this condition. Presenting symptoms of CGI are postprandial and exerciserelated pain, weight loss, and malabsorption [1]. Patients referred for evaluation of possible CGI are usually evaluated with computed tomography angiography (CTA) or magnetic resonance angiography (MRA). In a few expert centers, these angiographic methods are combined with gastrointestinal tonometry (TM), a functional test for mucosal saturation [2]. CTA and MRA are minimally invasive techniques to detect and define abdominal artery stenoses, while TM is a functional test that measures tissue ischemia. However, TM is an invasive and cumbersome procedure to perform, which explains the very limited use of this technique. Serum markers for CGI would be of great additional value as a noninvasive diagnostic test method.

In acute GI ischemia, several 'late' serum markers like lactate dehydrogenase (LDH), leucocyte counts, C-reactive protein (CRP) and L-lactate have been shown to have predictive value [3-5]. In the same patient group, 'early' serum markers like intestinal fatty acid binding protein (I-FABP), D-dimer and citrulline, have already been shown to be of value in diagnosing acute GI ischemia at an early stage [3, 610]. In contrast to acute GI ischemia, chronic GI ischemia is often reversible and usually limited to certain provocating factors, such as during exercise or after a meal. The value of 'early' serum markers to diagnose CGI patients is currently unknown. A recent study suggested a possible relationship between serum I-FABP and transient postprandial mucosal ischemia detected with TM in CGI patients [7]. D-dimer is a fibrinolytic marker of acute vascular events. It has been described as an early marker for acute mesenteric ischemia [11-15]. Small-bowel function might be altered in CGI patients due to hypoperfusion of the small bowel region caused by compromised blood flow in the superior mesenteric artery. Therefore, malabsorption syndrome and unexplained diarrhea may be the initial or the dominant feature of the clinical presentation in these patients [16]. Furthermore, since enterocytes are the predominant generators of serum citrulline, we hypothesized that citrulline could act as marker of intestinal mucosal injury in patients with CGI [17].

This study was performed to establish the potential diagnostic role of 'early' serum markers of GI ischemia and intestinal mucosal injury in patients suspected of CGI.

\section{Methods}

Consecutive patients referred for evaluation of possible CGI were asked to participate and were prospectively included after informed consent was obtained. The study was approved by the Institutional Review Board of the Erasmus MC University Medical Center.

\section{Diagnostic Work-Up}

In all patients, more common causes of chronic abdominal symptoms had been previously excluded by appropriate diagnostic evaluation. Medication use, including the use of platelet aggregation inhibitors, was scored in all participants. All patients were evaluated with our multidisciplinary CGI algorithm using our previously published protocol, which consists of TM combined with either CTA or MRA to visualize the celiac artery (CA), superior mesenteric artery (SMA) and inferior mesenteric artery (IMA)). A significant stenosis of the abdominal arteries was defined as a luminal obstruction of $>70 \%$. The findings from the assessment including medical history, symptom description, and the results of all diagnostic procedures were discussed in a multidisciplinary team consisting of a vascular surgeon, intervention radiologist, and gastroenterologist. A consensus diagnosis was made with one of the following conclusions: (1) no gastrointestinal stenosis, no ischemia, (2) gastrointestinal stenosis, no ischemia, (3) non-occlusive mesenteric ischemia (NOMI) (4) gastrointestinal stenosis and ischemia (i.e., CGI). Patients with CGI were offered revascularization of the vascular obstruction by either open surgery or by endovascular stent placement. A definitive diagnosis of CGI was confirmed after persistent relief of symptoms on post-treatment follow-up.

\section{Twenty-Four Hour Gastric and Jejunal Tonometry}

TM was performed to obtain mucosal $\mathrm{CO}_{2}$ measurements, both in fasting and postprandial states with gastric and jejunal catheters. All patients had meals at standard times during TM: liquid compound meal (12:00 PM), bread meal (6:00 PM), breakfast (8:00 AM), liquid compound meal (10:00 AM) and dinner (12:00 PM) [18]. The liquid compound meal consisted of two Nutridrink (Nutricia, Zoetermeer, The Netherlands) 200-ml packages. The patients were instructed to eat their meals in $15 \mathrm{~min}$. The cut-off values for elevated mucosal $\mathrm{CO}_{2}$ levels, as evidence of mucosal ischemia, were a gastric or jejunal $\mathrm{PCO}_{2}>12.0 \mathrm{kPa}$ after breakfast or a bread meal, $>13.6 \mathrm{kPa}$ after dinner, or $>10.6 \mathrm{kPa}$ after ingestion of a compound solution. The criteria for a positive test (abnormal result) on TM were: pathologic responses after three or more meals, or a combination of one or two pathologic responses after meals combined with a median $\mathrm{PCO}_{2}>8.0 \mathrm{kPa}$ measured in between meals. This was all done according to previously published standards [2]. 
Healthy Subjects

In all healthy subjects, abdominal artery stenosis was excluded by duplex ultrasound after fasting for $6 \mathrm{~h}$. Subsequently, blood samples were drawn at baseline and then at 30,60, 120, and 240 min after a standard liquid compound meal. No TM was performed in the healthy subjects.

\section{Blood Samples Serum Markers}

During TM, blood samples for analysis of I-FABP and D-dimer were drawn at a baseline fasting state and at 30,60, 120, and $240 \mathrm{~min}$ after the first standard liquid compound meal [18]. Blood samples for analysis of LDH, leucocyte counts, CRP and L-lactate were drawn at baseline and at 60 and $240 \mathrm{~min}$ after the first standard liquid compound meal.

For I-FABP analysis, blood samples were collected in SST tubes at room temperature. The serum was separated by centrifugation and stored at $-70^{\circ} \mathrm{C}$ until the assay was performed. Serum I-FABP was measured in duplicate at room temperature using enzyme-linked immunosorbent assay (Human I-FABP ELISA test kit, Hycult biotechnology B.V., Uden, The Netherlands). This kit has a minimum detection level of $0.02 \mu \mathrm{g} / \mathrm{l}$ and the I-FABP level was defined as abnormal above $0.1 \mu \mathrm{g} / \mathrm{l}$.

For D-dimer analysis, blood samples were collected in citrate tubes at room temperature. D-dimer concentration was determined using a quantitative immunofiltration assay method (D-Dimer VIDAS, BioMérieux, France). A D-dimer level above a cut-off of $0.50 \mathrm{mg} / \mathrm{l}$ was considered abnormal.

For LDH and CRP analysis, blood samples were collected in SST tubes at room temperature. LDH was determined using a quantitative in vitro test on Roche automated clinical chemistry analyzers (LDH, Modular P analyzer, Roche, Almere, The Netherlands). The LDH cutoff level was 449 U/l. CRP concentration was determined using a quantitative immunoturbidimetric assay on Roche automated clinical chemistry analyzer (CRPLX, Modular P analyzer, Roche, Almere, the Netherlands). The CRP cutoff level was $9 \mathrm{mg} / \mathrm{l}$.

For leucocyte count analysis, blood samples were collected in EDTA tubes at room temperature. Leucocyte counts were determined using a flowcytometric assay on Sysmex XE-2100 (WBC, Sysmex Se-2100, Goffin Meyvis, Etten-Leur, The Netherlands). The reference range for leucocyte counts was $3.5-10.0 * 10^{9} / 1$.

For L-lactate analysis, blood samples were collected in heparin tubes at room temperature. L-lactate was determined using an electrochemical assay on ABL 825 (L-lactate, ABL 825, Radiometer, Copenhagen, Denmark). The reference range for L-lactate concentrations was $0.5-1.7 \mathrm{mmol} / \mathrm{l}$. False L-lactate elevations due to peripheral vein sampling with venous stasis was avoided by using an intravenous catheter.

\section{Small-Bowel Function Tests}

After a fasting period of $6 \mathrm{~h}$, a sugar absorption test (SAT) was performed, consisting of an enzymatic measurement of mannitol/raffinose/sucrose/lactose in a 5-h urine sample. The reference range for the raffinose/mannitol ratio was 5-20. Also at baseline, blood samples for determination of citrulline and its precursor glutamine and its resultant arginine were collected in EDTA tubes at room temperature. Plasma was isolated by centrifugation at $2,650 \times g_{\max }$ for $10 \mathrm{~min}$ at $20^{\circ} \mathrm{C}$, and samples were stored at $-80^{\circ} \mathrm{C}$ until assay. Plasma was deproteinized with 5-sulphosalicylic acid $(6 \%, \mathrm{w} / \mathrm{v})$ containing norvaline and homoserine as internal standards. Amino acids were assayed by high-performance liquid chromatography (HPLC) using automated precolumn derivatization with o-phthaldialdehyde and fluorescence detection [19]. The normal references for fasting plasma glutamine, citrulline and arginine concentrations were 432-726, 18-47, and 26-107 $\mu \mathrm{mol} / \mathrm{l}$, respectively.

\section{Statistical Analysis}

Data were expressed as mean (range). The means were compared using the Mann-Whitney $U$ test at all different times of measurement. The D-dimer levels measured at subsequent points in time were compared using the Wilcoxon signed-rank test. Statistical analysis was performed using the SPSS 16.0 program (SPSS Inc. Chicago, IL, USA). Because serum marker measurements in subsequent blood samples of the same subject were correlated, the association between the levels of serum markers and the diagnosis chronic gastrointestinal ischemia was examined by means of linear regression repeated measures analyses as implemented in PROC MIXED (Statistical Analysis System). A $p$ value $<0.05$ was considered statistically significant (all two-tailed). To correct for multiple testing, a Bonferroni correction was used (five tests $p<0.010$, three tests $p<0.017)$.

\section{Results}

During a period of 5 months, October 2008-April 2009, 49 patients were referred for evaluation of possible CGI to our tertiary referral center with a dedicated CGI program. Informed consent for participation in the study was obtained from 42 patients and ten healthy participants. In two patients and one healthy participant, blood sample collections were incomplete due to venous catheter problems; they were consequently excluded from further 
Table 1 Patient characteristics and presenting symptoms, data given are number of patients (percentages) or mean (range)

\begin{tabular}{ll}
\hline & $n=40$ \\
\hline Age (years) & $60(20-86)$ \\
Gender M/F & $24 / 16$ \\
Postprandial pain & $29(73 \%)$ \\
Exercise related pain & $24(60 \%)$ \\
Diarrhea & $8(20 \%)$ \\
Weight loss & $25(63 \%)$ \\
Weight loss (kg) & $13(4-22)$ \\
BMI (kg/m ${ }^{2}$ ) & $22.4(15.0-37.3)$ \\
Abdominal symptoms & \\
Duration of symptoms (months) & $37(93 \%)$ \\
Risk factors for cardiovascular disease & $22(2-180)$ \\
- Smoking & \\
- Other risk factors & \\
Ischemia & $19(48 \%)$ \\
- Single-vessel stenosis & $31(78 \%)$ \\
- Multi-vessel stenosis & $32(80 \%)$ \\
- Non-occlusive mesenteric ischemia & 18 \\
Platelet aggregation inhibitor use & 4 \\
- Ischemia patients & 10 \\
- Non-ischemia patients & \\
\hline
\end{tabular}

${ }^{a}$ Including postprandial pain, exercise related pain and diarrhea

b Including diabetes mellitus, obesity, hypertension, hyperlipedemia, hyperhomocysteinemia, and familial history for cardiovascular disease

evaluation. CGI was diagnosed in 32 patients (80\%). Patient characteristics and presenting symptoms are presented in Table 1.

\section{Healthy Subjects}

Duplex scanning of the abdominal arteries showed normal flow patterns in the CA and SMA in all healthy participants. The serum levels of I-FABP remained undetectable, the serum levels of glutamine, citrulline, arginine, leucocyte counts, and L-lactate remained within normal range and the serum levels of D-dimer, LDH and CRP remained low before and after the standard test meal in all healthy participants. No healthy participants reported use of platelet aggregation inhibitors.

\section{Early Serum Markers: Intestinal Fatty Acid Binding Protein and D-dimer}

The serum levels of I-FABP remained undetectable in nonischemia patients at all different times of measurement. The serum levels of I-FABP were elevated in four patients diagnosed with ischemia; in three patients at all different times of measurement and in one patient at baseline and
Table 2 Levels of early serum markers in ischemia $(n=32)$ and non-ischemia $(n=8)$ patients (mean)

\begin{tabular}{lcrrrr}
\hline & Baseline & $30 \mathrm{~min}$ & $60 \mathrm{~min}$ & $120 \mathrm{~min}$ & $240 \mathrm{~min}$ \\
\hline I-FABP $(\mu \mathrm{g} / \mathrm{l})$ & & & & & \\
Ischemia & 0.06 & 0.05 & 0.05 & 0.05 & 0.03 \\
Non-ischemia & $<0.02$ & $<0.02$ & $<0.02$ & $<0.02$ & $<0.02$ \\
D-dimer (mg/l) & & & & & \\
Ischemia & 0.72 & 0.87 & 0.73 & 0.80 & 0.80 \\
Non-ischemia & 0.44 & 0.47 & 0.46 & 0.46 & 0.48 \\
\hline
\end{tabular}

Blood samples of both I-FABP and D-dimer were drawn at baseline and $30,60,120$, and 240 min after a standard meal

30 min after a meal. The levels did not differ between patients with and without ischemia at all different times of measurement (Table 2).

The mean D-dimer levels were elevated in ischemia patients and remained low in patients without ischemia. A non-significant D-dimer elevation of $0.33 \quad(95 \%$ CI $0.15-0.80) \mathrm{mg} / \mathrm{l}$ was seen in ischemia patients as compared to non-ischemia patients $(p=0.17)$. These levels did not differ between patients with and without ischemia at all different times of measurement (Table 2). Nevertheless, comparing D-dimer levels in patients diagnosed with ischemia at baseline and $30 \mathrm{~min}$ after the standard meal, a significant $(p<0.01)$ elevation in D-dimer levels was shown. Comparison of 30- and 60-min measurements demonstrated a significant decrease in D-dimer levels in these patients $(p<0.01)$ (Fig. 1). The sensitivity and specificity of the postprandial elevation of D-dimer were both $63 \%$.

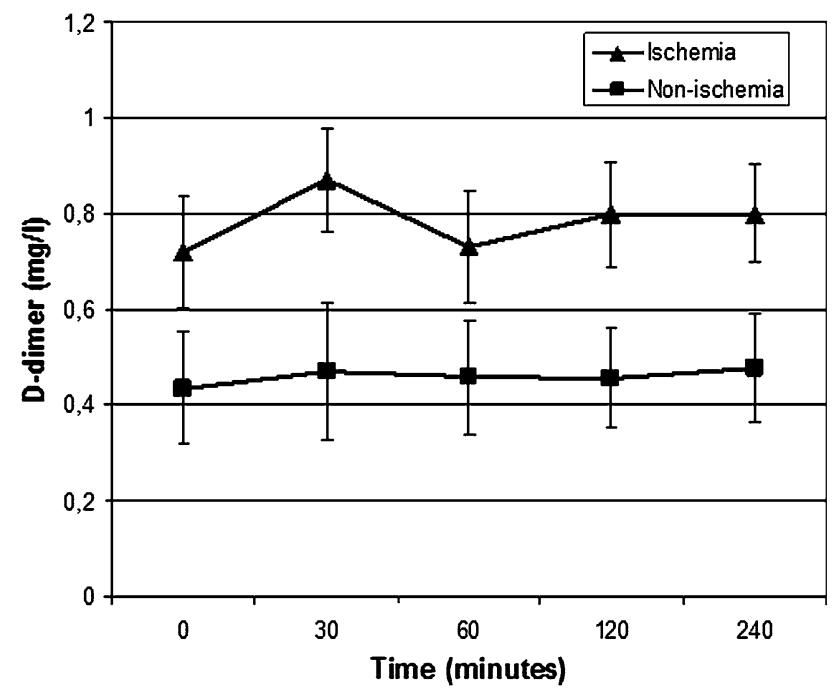

Fig. 1 Mean levels of D-dimer with 95\% confidence intervals in ischemia $(n=32)$ and non-ischemia $(n=8)$ patients at baseline and $30,60,120$, and $240 \mathrm{~min}$ after a meal. Ischemia patients: significant elevation at baseline 30 -min interval $(p=0.00)$, significant decrease at 30 to 60 -min interval $(p=0.00)$ 
Table 3 Levels of late serum markers in ischemia $(n=32)$ and non-ischemia $(n=8)$ patients (mean with range)
Blood samples of LDH, leucocyte counts, CRP and L-lactate were drawn at baseline, 60 and $240 \mathrm{~min}$ after a standard meal

\begin{tabular}{|c|c|c|c|}
\hline & Baseline & $60 \mathrm{~min}$ & $240 \mathrm{~min}$ \\
\hline \multicolumn{4}{|l|}{ LDH (U/l) } \\
\hline Ischemia & $333(204-633)$ & $363(203-721)$ & $319(204-546)$ \\
\hline Non-ischemia & $332(272-424)$ & $310(202-369)$ & $320(216-446)$ \\
\hline \multicolumn{4}{|c|}{ Leucocyte counts $\left(* 10^{9} / \mathrm{l}\right)$} \\
\hline Ischemia & $6.7(2.7-12.5)$ & $7.3(2.5-12.5)$ & $7.7(2.6-13.9)$ \\
\hline Non-ischemia & $5.6(3.9-8.8)$ & $5.8(3.7-9.0)$ & $6.3(4.5-9.2)$ \\
\hline \multicolumn{4}{|l|}{ CRP (mg/l) } \\
\hline Ischemia & $7(1-113)$ & $7(1-108)$ & $6(1-98)$ \\
\hline Non-ischemia & $17(1-67)$ & $18(1-68)$ & $17(1-67)$ \\
\hline \multicolumn{4}{|c|}{ L-lactate (mmol/l) } \\
\hline Ischemia & $1.4(0.8-2.4)$ & $1.7(1.0-2.9)$ & $1.3(0.6-2.3)$ \\
\hline Non-ischemia & $1.1(0.7-1.3)$ & $1.4(1.0-2.0)$ & $1.0(0.6-1.3)$ \\
\hline
\end{tabular}

Late Serum Markers: LDH, Leucocyte Counts, CRP, L-lactate

The LDH and CRP levels remained low and did not differ between patients with and without ischemia at all different times of measurement. The leucocyte counts remained within normal range and did not differ between patients with and without ischemia at all different times of measurement (Table 3). A significant L-lactate elevation of $0.29(95 \%$ CI $0.10-0.49) \mathrm{mmol} / 1$ was seen in ischemia patients as compared to non-ischemia patients $(p<0.01)$ (Fig. 2). The sensitivity and specificity of postprandial elevation of L-lactate were 34 and $88 \%$, respectively.

Intestinal Mucosal Injury

The SAT, glutamine, citrulline and arginine levels remained within normal range and did not differ between patients with and without ischemia (Table 4).

\section{Discussion}

In patients clinically suspected of CGI, the early serum markers I-FABP and D-dimer and the late serum markers LDH, leucocyte counts, and CRP were not different between patients with and without ischemia. Only L-lactate showed a significant elevation in ischemia patients compared to non-ischemia patients. Furthermore, in ischemia patients postprandial D-dimer levels were significantly higher compared to the fasting state. Small-bowel function in ischemia patients, as assessed with citrulline and compared to SAT, was within normal range and therefore intestinal function seems unaffected in these patients.

Until now, only one study has prospectively described increased I-FABP levels, indicating epithelial damage in patients with transient and reversible gastrointestinal

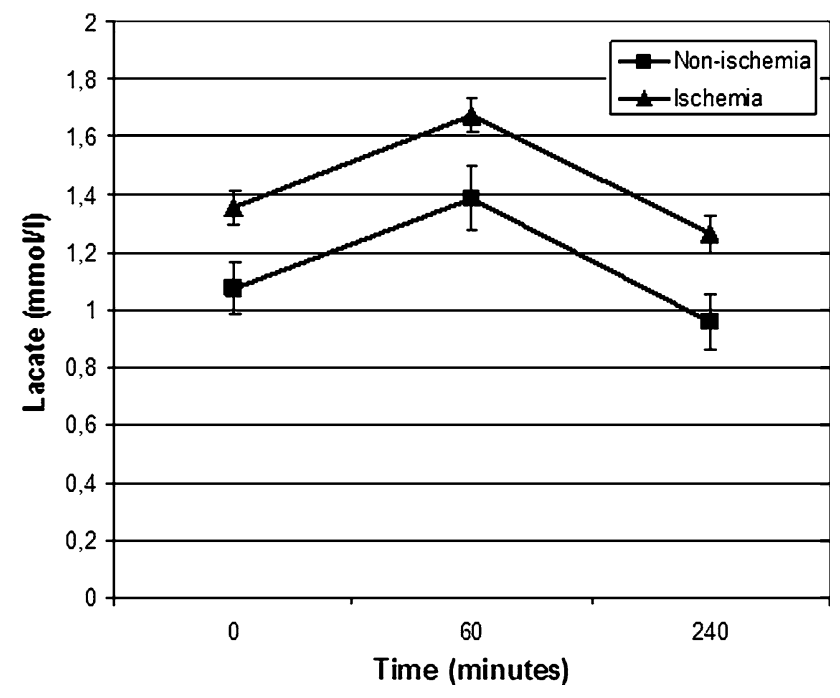

Fig. 2 Mean levels of L-lactate with $95 \%$ confidence intervals in ischemia $(n=32)$ and non-ischemia $(n=8)$ patients at baseline and 60 and $240 \mathrm{~min}$ after a meal. Ischemia patients compared to nonischemia patients: significant L-lactate elevation $(p=0.00)$

Table 4 Levels of serum markers in ischemia $(n=32)$ and nonischemia $(n=8)$ patients (mean with range)

\begin{tabular}{lc}
\hline Glutamine $(\mu \mathrm{mol} / \mathrm{l})$ & $598(461-821)$ \\
Ischemia & $582(407-678)$ \\
Non-ischemia & \\
Citrulline $(\mu \mathrm{mol} / \mathrm{l})$ & $31(20-48)$ \\
Ischemia & $30(25-38)$ \\
Non-ischemia & \\
Arginine $(\mu \mathrm{mol} / \mathrm{l})$ & $65(32-122)$ \\
Ischemia & $61(40-100)$ \\
Non-ischemia & \\
SAT (Raffinose/Mannitol ratio) & $9(3-20)$ \\
Ischemia & $10(4-20)$ \\
Non-ischemia & \\
\hline
\end{tabular}

Blood samples of glutamine, citrulline and arginine were drawn at baseline. SAT was performed in a urine sample at baseline 
mucosal ischemia, as detected with gastrointestinal TM [7]. In that study, blood samples were drawn at baseline and at 60,120 , and 240 min after a test meal. However, the halflife of I-FABP in humans is $22 \mathrm{~min}$ and therefore the maximal peak I-FABP levels may have been missed. Our study adjusted the timeline of blood sampling by adding a 30-min blood sample to the protocol in order to optimize the timing of measurements. Nevertheless, the results of the previous study were not reproducible. The serum levels of I-FABP in the current study remained undetectable in non-ischemia patients and were only elevated in four patients diagnosed with ischemia, and these levels did not differ between patients with and without ischemia. However, the fact that a rise in I-FABP levels was only found in CGI patients is striking, suggesting I-FABP might be of interest as a possible diagnostic tool to confirm the diagnosis of CGI. On the contrary, the expected prevalence of CGI in the patient group examined would be high (at least $>40 \%$ ) so a test, with a high specificity but poor sensitivity, may be less useful. Furthermore, a sample size of greater than 800 patients suspected of CGI would be needed to demonstrate a statistically significant difference using our I-FABP findings, which is unlikely to happen considering the low incidence of this disease entity. Therefore, it would be difficult to ever demonstrate a statistically significant difference using this test.

It is thought that in CGI patients, transient mucosal ischemia only occurs in response to increased metabolic demand, such as after a meal or exercise. In the current study, patients diagnosed with ischemia showed a significant postprandial rise in D-dimer levels as compared to the fasting state (Fig. 1). However, our observation of (nonsignificant) increased baseline levels of D-dimer in patients diagnosed with ischemia might suggest impaired mucosal perfusion during resting conditions as well. The lack of a statistically significant difference in the elevation of D-dimer levels in ischemia patients as compared to the normal D-dimer levels in patients without ischemia could possibly be due to insufficient sample size to achieve sufficient power to demonstrate this effect (Fig. 1). Several earlier studies that investigated the use of D-dimer in acute gastrointestinal ischemia showed that an elevated D-dimer level had a high sensitivity, but lacked specificity $[3,15]$. D-dimer measurements can be routinely determined in every hospital laboratory and therefore could be a useful tool contributing to the diagnosis CGI.

Plasma L-lactate has been advocated as a promising marker of acute gastrointestinal ischemia in human studies $[20,21]$. However, experimental studies in pigs suggest that peripheral plasma L-lactate is not a useful early marker $[5,22]$. In our study, the time course of L-lactate concentration showed a significant elevation in chronic ischemia patients compared to non-ischemia patients. This is in contrast to the theory that the capacity of the liver to clear large quantities of L-lactate from the porto-mesenteric circulation may prevent systemic L-lactate elevations [23]. Since L-lactate is considered to be a marker for the more advanced phase of acute abdominal disorders, it was thought to not be a relevant marker in the early transient stages of gastrointestinal ischemia. However, in the current study, chronic ischemia patients showed elevated L-lactate levels after meal-provocation, which were also significantly increased in ischemia patients as compared to nonischemia patients, suggesting that L-lactate levels could indeed be used as a possible early marker. However, L-lactate is often found in non-gastrointestinal disorders, such as shock, septicemia, hepatic and renal failure, and diabetic ketoacidosis and therefore, is not specific to CGI. Despite this limitation, the change in pre- and postprandial L-lactate measurements could be a useful supplementary diagnostic test contributing to the diagnosis CGI. The major advantage of L-lactate measurements is that it can be measured easily in any hospital laboratory and provides a rapid result.

Citrulline is thought to detect decreased intestinal absorption, which is regarded as a marker of intestinal failure. The role of citrulline as a biomarker has been previously investigated in patients with short bowel syndrome, celiac disease, intestinal graft versus host disease and radiation-induced small bowel injury, showing promising results [17, 24-27]. Since bowel function is thought to be altered in CGI, citrulline was measured to assess intestinal mucosal injury in patients diagnosed with ischemia and compared with SAT, which is the gold standard for bowel function. The time required to achieve abnormal citrulline levels after a reversible ischemic event is a matter of days. Therefore citrulline and its precursor glutamine and its resultant arginine were only tested at baseline to evaluate the bowel function in CGI patients. Both the levels of the index test and the reference standard remained within the normal range and did not differ between ischemia and non-ischemia patients. As a result, intestinal function seems to be preserved in patients diagnosed with ischemia.

A possible limitation of the study is the relatively small number of patients without ischemia compared to the large number of patients diagnosed with ischemia. However, serum levels of all early and late markers showed normal values in the non-ischemia patients. Therefore, this population seems to be similar to the "normal population." A second limitation is the use of platelet aggregation inhibitors, which could lead to possible bias. One study reported elevated I-FABP levels in a healthy participant after the use of NSAIDs [7]. Platelet aggregation inhibitor use, including "over the counter" use of NSAIDs, was reported by 12 $(38 \%)$ ischemia patients and four $(50 \%)$ non-ischemia- 
patients, and in $50 \%$ of the ischemia patients with elevated I-FABP levels in this study. However, higher I-FABP levels in patients using platelet aggregation inhibitors were not seen as compared to non-users, after statistical correction for the use of these drugs.

In conclusion, I-FABP, leucocyte counts, LDH and CRP levels did not differ in patients with and without CGI, both before and after a test meal, and therefore do not seem to be clinically useful for the diagnosis CGI. Small-bowel function appears not to be affected in patients diagnosed with ischemia. L-lactate and D-dimer serum levels, both before and after provocation, seem to be promising early indicators of mucosal ischemia. Future studies are necessary to investigate the use of L-lactate and D-dimer levels in the diagnosis of CGI.

Acknowledgments The Dutch Society of Gastroenterology is gratefully acknowledged for the "Gastrostart" allowance. Furthermore, C. Teshima, MD, is gratefully acknowledged for his assistance in preparing the manuscript.

Conflicts of interest The authors disclose no conflicts.

Funding D. van Noord received unrestricted grants from JanssenCilag, the Netherlands and the Dutch Society of Gastroenterology.

Open Access This article is distributed under the terms of the Creative Commons Attribution Noncommercial License which permits any noncommercial use, distribution, and reproduction in any medium, provided the original author(s) and source are credited.

\section{References}

1. Kolkman JJ, Bargeman M, Huisman AB, Geelkerken RH. Diagnosis and management of splanchnic ischemia. World $J$ Gastroenterol. 2008;14:7309-7320.

2. Mensink PB, Geelkerken RH, Huisman AB, Kuipers EJ, Kolkman JJ. Twenty-four hour tonometry in patients suspected of chronic gastrointestinal ischemia. Dig Dis Sci. 2008;53:133-139.

3. Block T, Nilsson TK, Bjorck M, Acosta S. Diagnostic accuracy of plasma biomarkers for intestinal ischaemia. Scand J Clin Lab Invest. 2008;68:242-248.

4. El-Awady SI, El-Nagar M, El-Dakar M, Ragab M, Elnady G. Bacterial translocation in an experimental intestinal obstruction model. C-reactive protein reliability? Acta Cir Bras. 2009;24: 98-106.

5. Kurimoto Y, Kawaharada N, Ito T, Morikawa M, Higami T, Asai Y. An experimental evaluation of the lactate concentration following mesenteric ischemia. Surg Today. 2008;38:926-930.

6. Kanda T, Fujii H, Fujita M, Sakai Y, Ono T, Hatakeyama K. Intestinal fatty acid binding protein is available for diagnosis of intestinal ischaemia: immunochemical analysis of two patients with ischaemic intestinal diseases. Gut. 1995;36:788-791.

7. Mensink PB, Hol L, Borghuis-Koertshuis N, et al. Transient postprandial ischemia is associated with increased intestinal fatty acid binding protein in patients with chronic gastrointestinal ischemia. Eur J Gastroenterol Hepatol. 2009;21:278-282.
8. Pelsers MM, Namiot Z, Kisielewski W, et al. Intestinal-type and liver-type fatty acid-binding protein in the intestine. Tissue distribution and clinical utility. Clin Biochem. 2003;36:529-535.

9. Cronk DR, Houseworth TP, Cuadrado DG, Herbert GS, McNutt PM, Azarow KS. Intestinal fatty acid binding protein (I-FABP) for the detection of strangulated mechanical small bowel obstruction. Curr Surg. 2006;63:322-325.

10. Peters JH, Wierdsma NJ, Teerlink T, van Leeuwen PA, Mulder CJ, van Bodegraven AA. Poor diagnostic accuracy of a single fasting plasma citrulline concentration to assess intestinal energy absorption capacity. Am J Gastroenterol. 2007;102:2814-2819.

11. Altinyollar H, Boyabatli M, Berberoglu U. D-dimer as a marker for early diagnosis of acute mesenteric ischemia. Thromb Res. 2006; 117:463-467.

12. Acosta S, Nilsson TK, Bjorck M. D-dimer testing in patients with suspected acute thromboembolic occlusion of the superior mesenteric artery. Br J Surg. 2004;91:991-994.

13. Acosta S, Nilsson TK, Bjorck M. Preliminary study of D-dimer as a possible marker of acute bowel ischaemia. $\mathrm{Br} J$ Surg. 2001;88:385-388.

14. Block T, Nilsson TK, Bjorck M, Acosta S. Diagnostic accuracy of plasma biomarkers for intestinal ischaemia. Scand J Clin Lab Invest. 2007;1-9.

15. Icoz G, Makay O, Sozbilen M, et al. Is D-dimer a predictor of strangulated intestinal hernia? World J Surg. 2006;30:2165-2169.

16. Meyers MA, Kaplowitz N, Bloom AA. Malabsorption secondary to mesenteric ischemia. Am J Roentgenol Radium Ther Nucl Med. 1973;119:352-358.

17. Curis E, Crenn P, Cynober L. Citrulline and the gut. Curr Opin Clin Nutr Metab Care. 2007;10:620-626.

18. Mensink PB, Geelkerken RH, Huisman AB, Kuipers EJ, Kolkman JJ. Effect of various test meals on gastric and jejunal carbon dioxide: a study in healthy subjects. Scand $J$ Gastroenterol. 2006;41:1290-1298.

19. Fekkes D, van Dalen A, Edelman M, Voskuilen A. Validation of the determination of amino acids in plasma by high-performance liquid chromatography using automated pre-column derivatization with o-phthaldialdehyde. J Chromatogr. 1995;669:177-186.

20. Lange $\mathrm{H}$, Toivola $\mathrm{A}$. [warning signals in acute abdominal disorders. Lactate is the best marker of mesenteric ischemia]. Lakartidningen. 1997;94:1893-1896.

21. Janda A, Hagmuller GW, Denck H. [lactate in the diagnosis of acute intestinal vascular occlusions]. Der Chirurg; Zeitschrift fur alle Gebiete der operativen Medizen. 1984;55:469-473.

22. Acosta S, Nilsson TK, Malina J, Malina M. L-lactate after embolization of the superior mesenteric artery. J Surg Res. 2007; 143:320-328.

23. Jakob SM, Merasto-Minkkinen M, Tenhunen JJ, Heino A, Alhava E, Takala J. Prevention of systemic hyperlactatemia during splanchnic ischemia. Shock Augusta, Ga. 2000;14:123-127.

24. Gondolesi G, Ghirardo S, Raymond K, et al. The value of plasma citrulline to predict mucosal injury in intestinal allografts. Am J Transplant. 2006;6:2786-2790.

25. Pappas PA, Tzakis AG, Gaynor JJ, et al. An analysis of the association between serum citrulline and acute rejection among 26 recipients of intestinal transplant. Am J Transplant. 2004;4: 1124-1132.

26. Pappas PA, Tzakis AG, Saudubray JM, et al. Trends in serum citrulline and acute rejection among recipients of small bowel transplants. Transplant Proc. 2004;36:345-347.

27. Lutgens L, Lambin P. Biomarkers for radiation-induced small bowel epithelial damage: an emerging role for plasma citrulline. World J Gastroenterol. 2007;13:3033-3042. 